\title{
Algebras for Combinatorial Search
}

\author{
Michael Spivey \\ Oxford University Computing Laboratory \\ Wolfson Building, Parks Road, Oxford OX1 3QD, UK \\ mike@comlab.ox.ac.uk
}

\begin{abstract}
We show how combinatorial search strategies including depth-first, breadth-first and depth-bounded search can be viewed as different implementations of a common algebraic specification that emphasises the compositionality of the strategies. This specification is placed in a categorical setting that combines algebraic specifications and monads.
\end{abstract}

Keywords: Functional Programming, Combinatorial Search, Algebra, Categories

\section{INTRODUCTION}

Many combinatorial algorithms can be expressed by forming a tree of choices, then searching it for solutions. In functional programming, we can formulate such problems neatly by composing a function that produces the tree with a function that searches the tree and produces (say) a list of solutions found in it:

$$
\begin{aligned}
& \text { solutions }:: \text { Input } \rightarrow[\text { Output }] \\
& \text { solutions } x=\text { search }(\text { make_tree } x)
\end{aligned}
$$

where

$$
\begin{aligned}
& \text { make_tree }:: \text { Input } \rightarrow \text { Tree Output } \\
& \text { search }:: \text { Tree } \alpha \rightarrow[\alpha],
\end{aligned}
$$

for some suitable type Tree $\alpha$ of trees. To be concrete, we could use for search a function that does depth-first search of the tree. As every functional programmer knows, this program works well under lazy evaluation, because it is not necessary to form the whole search tree before starting to search it, and it is not necessary to search the whole tree before producing the first item in the list of outputs. These observations become especially important if the search tree is infinite, because then lazy evaluation can produce some useful output even though the program can never terminate.

If the function make_tree describes a sequence of choices, then it can be written in a compositional way, so that the output of the first set of choices becomes the input to further choices later in the program. To make the compositional structure explicit, it is best to make the Tree type into a monad, so that there is an operation

$$
(\triangleright):: \text { Tree } \alpha \rightarrow(\alpha \rightarrow \text { Tree } \beta) \rightarrow \text { Tree } \beta
$$

(written $>>=$ in the ASCII syntax of Haskell). If $x t$ is a tree with leaves drawn from type $\alpha$, and $f$ is a function $\alpha \rightarrow$ Tree $\beta$, then $x t \triangleright f$ is another tree; its structure near the root mirrors the structure of $x t$, but each leaf $x$ of that tree has been replaced by the subtree $f x$, grafted in place of the leaf. All common varieties of tree can be made into monads in this way, but we will be particularly interested in 'rose trees', where a node may have a finite list of children of any length. We are using this monadic operation to model composition of non-deterministic programs, but it also has another interpretation: in an application where trees represent algebraic expressions, this operation implements substitution of expressions for the variables that appear in another expression. 
Now that we have given Tree $\alpha$ the structure of a monad, we can recall that the list type $[\alpha]$ is a monad too, and ask whether there is any relationship between the monad structure of trees and lists. The answer is a resounding "yes!": in fact the function search is a morphism of monads. Among other things, this means that the following algebraic law holds:

$$
\operatorname{search}(x t \triangleright f)=\operatorname{search} x t \triangleright^{\prime}(\lambda x \rightarrow \operatorname{search}(f x))
$$

where $\left(\triangleright^{\prime}\right)::[\alpha] \rightarrow(\alpha \rightarrow[\beta]) \rightarrow[\beta]$ is the monadic binding operator on lists. This law can be read as stating that depth-first search is compositional. That is, if all we want is the list of leaves of a tree in depth-first order, then there is no need to build the tree at all: we can replace a program $x t \triangleright f$ that builds a tree in two stages with the program $x s \triangleright^{\prime} f^{\prime}$, a composition of two programs $x s=$ search $x t$ and $f^{\prime} x=\operatorname{search}(f x)$ whose interfaces are in terms of lists of outputs, not trees of outputs. If the original $x t$ and $f$ were defined using further compositions of simpler programs, then we can hope to move the occurrences of search further in by applying the same law again, until we can finally eliminate search by replacing primitives that produce trees with their counterparts that produce lists.

These ideas raise a number of questions, which the present article aims to answer. First, what is an appropriate set of composition operators (in addition to $\triangleright$ ) for programs that produce search trees? Can the same operators be defined on lists so that the function search respects each operator as it does $(\triangleright)$ ?

Second, standard texts on Artificial Intelligence (see, e.g., [1]) describe other search algorithms like breadth-first search and depth-bounded search which are more effective that depth-first search, particularly on infinite trees. Can these search algorithms also be made compositional in the sense alluded to here?

Third, what is an appropriate algebraic setting in which to place these results? For standard collection types like sets, bags and lists, the Boom hierarchy [2], [3] provides a uniform setting within which similar programs can be developed in a similar way. Is there an analogous setting for the monads that are associated with non-deterministic search?

\section{COMBINATORS FOR SEARCH}

The first task is to find a set of combinators that allow us to build up search trees, with the hope that they can be implemented for the various search strategies in a way that makes them compositional. We will first define five operations that work with trees: zero, return, $\triangleright, \oplus$, and wrap, then look at the algebraic laws that they satisfy; in effect, this will give us an algebraic specification of search. Next, we will look for other data types that satisfy the same specification, corresponding to different search algorithms. We will show that the implementation based on trees is an initial object in a certain category, so that any other implementation can be viewed as compositional in the sense outlined in the Introduction.

Rather than operating directly on trees, our initial set of combinators will work on forests - that is, finite lists of trees - because this will allow us to define an associative or operator that combines two collections of results into one. Our trees will be 'rose trees', where each internal node has a finite list - a forest - of children.

$$
\begin{aligned}
& \text { data Tree } \alpha=\text { Leaf } \alpha \mid \text { Fork (Forest } \alpha) \\
& \text { type Forest } \alpha=[\text { Tree } \alpha] .
\end{aligned}
$$

There is an empty forest, which we will call zero, and given any value $x:: \alpha$, we can form the tiny forest return $x$ that contains just the leaf $x$ :

$$
\begin{aligned}
& \text { zero }:: \text { Forest } \alpha \\
& \text { zero }=[] \\
& \text { return }:: \alpha \rightarrow \text { Forest } \alpha \\
& \text { return } x=[\text { Leaf } x]
\end{aligned}
$$

Of course, return will become the unit of our monad of forests. 
Given two forest $x f$ and $y f$, we can concatenate them to form a wider forest $x f \oplus y f$. Also, we can wrap up any forest $x f$ as a new forest that contains a single tree; in this way we can obtain forests that are not just lists of leaves.

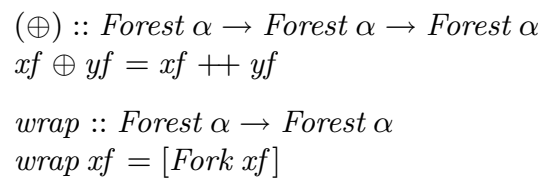

It is easy to see by an inductive argument that any forest can be constructed using the operations we have so far introduced.

The final operation in our suite of combinators is the binding operator $\triangleright$ that makes forests into a monad. As we shall see later, the definition of the this operator is more-or-less forced on us by algebraic considerations, but for now we can give a recursive definition as follows, in terms of a function bindt that is mutually recursive with $\triangleright$ :

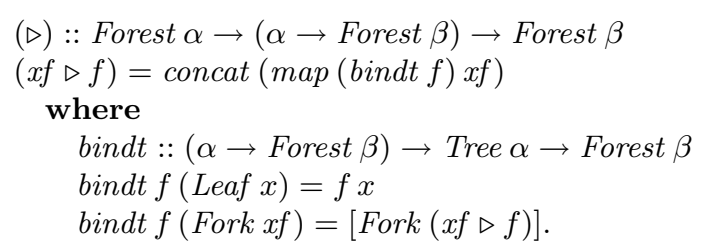

Now we turn to the algebraic laws that are satisfied by the operations we have introduced; these are important, because we will require that they should also hold for the other search strategies we wish to consider. First of all, we find that return and $\triangleright$ form a monad, so that the following laws apply:

$$
\begin{aligned}
(\text { return } x) \triangleright f & =f x, \\
x t \triangleright \text { return } & =x t, \\
(x t \triangleright f) \triangleright g & =x t \triangleright(\lambda x \rightarrow f x \triangleright g) .
\end{aligned}
$$

We also find that zero and $\oplus$ form a monoid:

$$
\begin{aligned}
(x t \oplus y t) \oplus z t & =x t \oplus(y t \oplus z t), \\
z e r o \oplus x t & =x t, \\
x t \oplus z \text { ero } & =x t .
\end{aligned}
$$

The monad and the monoid interact according to the following laws, which state that for any function $f:: \alpha \rightarrow \beta$, the function $(\triangleright f)::$ Forest $\alpha \rightarrow$ Forest $\beta$ is a homomorphism of monoids:

$$
\begin{aligned}
\text { zero } \triangleright f & =\text { zero, } \\
(x t \oplus y t) \triangleright f & =(x t \triangleright f) \oplus(y t \triangleright f) .
\end{aligned}
$$

Finally, wrap and $\triangleright$ are related by the law,

$$
(\text { wrap } x f) \triangleright f=\operatorname{wrap}(x f \triangleright f) .
$$

In fact, it is a straightforward induction, given the definitions of the other four operations, to verify that the definition of $\triangleright$ given above is the only one that satisfies the laws (1), (7), (8) and (9).

Now we define a bunch to be a type constructor $M$, together with five polymorphic operations,

$$
\begin{aligned}
& \text { return }:: M \alpha, \\
& (\triangleright):: \alpha \rightarrow(\alpha \rightarrow M \beta) \rightarrow M \beta, \\
& \text { zero }:: M \alpha, \\
& (\oplus):: M \alpha \rightarrow M \alpha \rightarrow M \alpha, \\
& \text { wrap }:: M \alpha \rightarrow M \alpha,
\end{aligned}
$$


such that the laws (1) to (9) are satisfied. As we shall see in the next section, several well-known search methods can be expressed as bunches.

We have observed in earlier work [4] that Haskell's constructor classes provide a good framework for programming different bunches, so that an entire program can be written in a way that is indifferent to the bunch that is to be used for its execution. We forbear to do this in the present paper, as it not needed for the exposition, and we can therefore also suppress the newtype construction that is needed in Haskell to allow instances of constructor classes to be associated with compound type constructors.

\section{EXAMPLES OF BUNCHES}

Depth-first search. To obtain a bunch that gives the effect of depth-first search, we can take $M \alpha$ to be the type of (lazy) streams over $\alpha$, and define the five operations as follows:

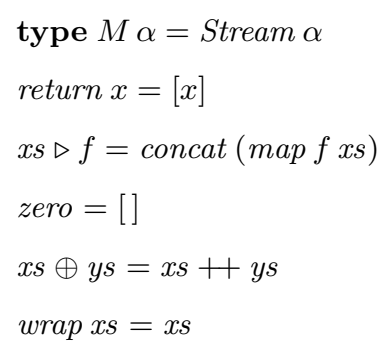

These definitions do in fact satisfy the nine laws needed for a bunch.

Breadth-first search. A bunch that gives the effect of breadth-first search can be based on the type of streams of bags over $\alpha$, with the idea that successive (finite) bags in the potentially infinite stream give the solutions from successive levels of the search tree. The stream is finite or infinite according to whether the search tree for the same problem is finite or infinite. The relevant definitions are (roughly) as follows:

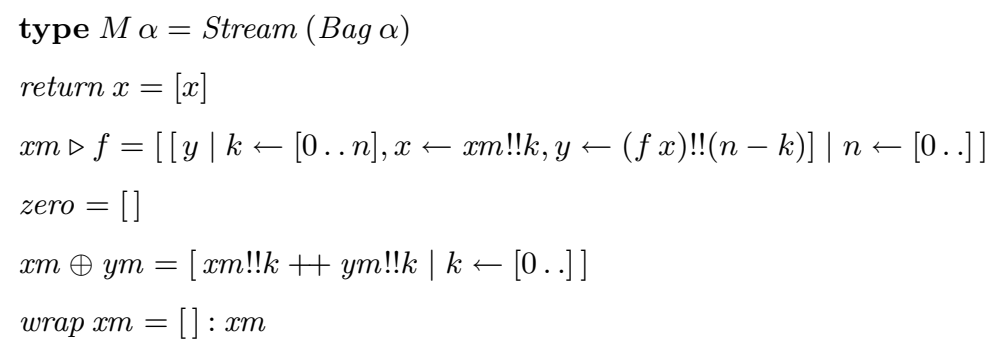

(We use list notation for both finite bags and infinite streams, but treat two lists as equal if they represent bags with the same elements in a different order.)

I used the word "roughly" because the definitions of $\triangleright$ and $\oplus$ given here assume that all streams are infinite, even if the program would produce a finite search tree; a better definition of $\oplus$, and one that avoids the inefficiency associated with the indexing operator !!, is as follows:

$x m \oplus y m=$ longzipwith $(+) x m$ ym

where longzipwith is a version of zipwith that works even when its arguments are lists of different lengths. When one list is exhausted, the remainder of the other list is copied over to the result:

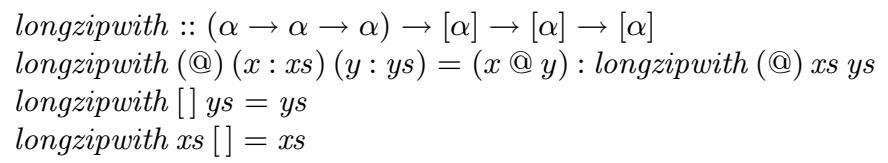

Obviously, longzipwith (@) is associative whenever (@) itself is associative, and longzipwith (@) has the empty list as a unit element. 
In addition to being restricted to infinite streams, and inefficiently using !!, the definition of $\triangleright$ given here suffers from a more serious inefficiency, in that the expression $f x$ may be evaluated many times for the same value of $x$. We can use equations (1), (7), (8) and (9) to derive a better definition of $\triangleright$ that copes with finite streams. Equation (7) gives us immediately that

$$
\text { [] } f=[] \text {. }
$$

Also if $x b=\left[x_{1}, \ldots, x_{n}\right]$ is any finite bag, then $x b$ can be written $x b=$ return $x_{1} \oplus \ldots \oplus$ return $x_{n}$, so a stream $x b: x b s$ can be written

$$
x b: x b s=\left(\text { return } x_{1} \oplus \ldots \oplus \text { return } x_{n}\right) \oplus \text { wrap xbs. }
$$

Applying equations (8), (1) and (9) to this, we obtain

$$
\begin{aligned}
(x b: x b s) \triangleright f & =\left(f x_{1} \oplus \ldots \oplus f x_{n}\right) \oplus \operatorname{wrap}(x b s \triangleright f) \\
& =\text { concatall }(\operatorname{map} f x b) \oplus \operatorname{wrap}(x b s \triangleright f) .
\end{aligned}
$$

where concatall $=$ foldr $(\oplus)$ zero. This yields the following definition of $\triangleright$ :

$$
\begin{aligned}
& {[] \triangleright f=[]} \\
& (x b:: x b s) \triangleright f=\text { concatall }(\operatorname{map} f x b) \oplus \operatorname{wrap}(x b s \triangleright f)
\end{aligned}
$$

We will discuss later in this paper why we can be sure that the operation defined by these equations satisfies all the laws needed to be a bunch.

As we explained in an earlier paper [4], and will later explore from a different point of view, it is necessary to use streams of bags, ignoring the order of elements in each layer, if the composition operator $\triangleright$ is to obey the associative law (3).

Depth-bounded search. Standard texts on artificial intelligence (for example, [1]) observe that breadth-first search typically has large memory requirements, and recommend depth-bounded search as a more efficient alternative. This is similar to depth-first search (and so can be implemented efficiently on a stack), but the search is made finite by cutting off all branches of the search tree at a given depth. In order to make the search complete, the technique of iterative deepening is used: this amounts to performing repeated searches with a depth bound that slowly increases. Naturally, this entails recomputing the shallow parts of the tree repeatedly, but in practice this is often faster than maintaining the data structure that would be needed (as in breadth-first search) to avoid the recomputation.

In order to make depth-bounded search compositional, we need to know the depth at which each answer is found, so we perform the search with a function that takes a depth bound and returns a list of answers, each paired with an integer:

type DBound $\alpha=$ Int $\rightarrow[(\alpha$, Int $)]$

The definitions come out simplest if we pair each answer with the unused portion of the bound: thus if the supplied bound is $n$, and the answer $x$ occurs at depth $d \leq n$, then the pair $(x, n-d)$ will appear among the results.

For consistency, we require that each answer that appears for a depth bound $n$ should continue to appear for each greater bound $n+k$. Thus if $p::$ DBound $\alpha$ and $k \geq 0$ then $p n$ contains $(x, r)$ if and only if $p(n+k)$ contains $(x, r+k)$. To implement iterative deepening, we simply need to form the list $[x \mid n \leftarrow[0 .],.(x, r) \leftarrow p n, r \equiv 0]$.

The basic operations return and $\triangleright$ are defined as follows:

$$
\begin{aligned}
& (\text { return } x) n=[(x, n)] \\
& (p \triangleright f) n=[(y, s) \mid(x, r) \leftarrow p n,(y, s) \leftarrow f x r] .
\end{aligned}
$$

These satisfy the equations needed for a monad; for example, the associative law (3) is satisfied because both $(p \triangleright f) \triangleright g$ and $p \triangleright(\lambda x \rightarrow f x \triangleright g)$ when applied to a bound $n$ yield the list

$$
[(z, t) \mid(x, r) \leftarrow p n,(y, s) \leftarrow f x r,(z, t) \leftarrow g y s] .
$$


The convention that pairs each solution with the remaining depth budget simplifies the details of both the definition of $\triangleright$ and the argument here.

The extra operations zero, $\oplus$ and wrap are equally straight-forward to define:

$$
\begin{aligned}
& \text { zero } n=[] \\
& \left(p_{1} \oplus p_{2}\right) n=p_{1} n++p_{2} n \\
& (\operatorname{wrap} p) 0=[] \\
& (\operatorname{wrap} p)(n+1)=p n
\end{aligned}
$$

The idea behind wrap is that the solutions with depth $\leq n+1$ in wrap $p$ should be the same as those with depth $\leq n$ in $p$; the remaining depth budget that is paired with each solution is unchanged.

The equation (8) is interesting, because it is not satisfied by a putative bunch based on the type constructor Maybe. It is straightforward to make this type constructor into a monad, and we can define an associative operation $\oplus$ by

$$
\begin{aligned}
& (\oplus):: \text { Maybe } \alpha \rightarrow \text { Maybe } \alpha \rightarrow \text { Maybe } \alpha \\
& \text { Nothing } \oplus \text { ym }=\text { ym } \\
& \text { Just } x \oplus \text { ym }=\text { Just } x
\end{aligned}
$$

However, these do not satisfy the equation. If even is a function such that even $2=J u s t 2$ and even $3=$ Nothing, then

$$
(\text { Just } 3 \oplus \text { Just } 2) \triangleright \text { even }=\text { Just } 3 \triangleright \text { even }=\text { Nothing, }
$$

but

$$
(\text { Just } 3 \triangleright \text { even }) \oplus(\text { Just } 2 \triangleright \text { even })=\text { Nothing } \oplus \text { Just } 2=\text { Just } 3,
$$

so the law does not hold. This reflects the fact that once a computation of type Maybe $\alpha$ has delivered a result, it is not possible to backtrack and obtain a different result if the first one causes the rest of the computation to fail. This means that the sort of non-backtracking failure represented by the Maybe type is not part of the family of search strategies being considered here, even though Maybe is made a member of the MonadPlus type class in Haskell's standard library.

Another non-example of our laws can be found in a recent paper by Kiselyov, Shan, Friedman and Sabry [6]. They attempt to enhance a stream-based model of backtracking with alternative combinators that implement fair interleaving (including bindi, a fair version of $\triangleright$ ), and they show how this interleaving can be realised in a continuation-passing style. As we have noted earlier [4], such an attempt is doomed to failure, because there is no way for a computation that returns a stream to signal to its environment that it has done some work but found no answers. Briefly, in the computation

$$
\text { do } a \leftarrow \text { intsfrom } 2 \text {; (do } b \leftarrow \text { intsfrom } 2 \text {; test }(a * b \equiv 9)),
$$

the inner computation (do $b \leftarrow \ldots$ ) necessarily diverges when applied to the value $a=2$, but the morally equivalent computation

$$
\begin{aligned}
& \text { do }(a, b) \leftarrow \text { interleave }(\text { intsfrom } 2)(\text { intsfrom } 2) ; \text { test }(a * b \equiv 9) \\
& \quad \text { where interleave } x m \text { ym }=\text { do } x \leftarrow x m ; y \leftarrow y m ; \operatorname{return}(x, y)
\end{aligned}
$$

does have a chance to interleave the two streams of integers fairly, and so can produce the answer $(3,3)$ - before also diverging in the hunt for other pairs that multiply to give 9 . The failure of these two expressions to be equivalent boils down to a failure of the bindi combinator to satisfy the associative law (3). This failure of associativity seems unimportant in a small example such as this, because it is no trouble to write the second of the two expressions in place of the first; but in a larger example, the two non-deterministic choices may be far apart in the text of a complex program, and it may be impossible to bring them together in order to make the interleaving fair without damaging the structure of the program. 


\section{A CATEGORICAL VIEW}

A more abstract view of the relationships between different search strategies can be obtained using concepts from category theory. We know that each strategy involves a monad, but a strategy is more than that, because it also contains the combining operators on results.

It may be helpful to give a reminder of how the notation for monads that is used in category theory is related to that used in functional programming. In programming, a monad consists of a type constructor $M$, together with polymorphic functions return :: $\alpha \rightarrow M \alpha$ and ( $\triangleright):: M \alpha \rightarrow$ $(\alpha \rightarrow M \beta) \rightarrow M \beta$. In the notation of category theory, each instance of return becomes an arrow $\eta_{x}: x \rightarrow M x$, and for each arrow $f: x \rightarrow M y$, we can form an arrow $f^{*}: M x \rightarrow M y$; this corresponds to the function that maps $x m:: M \alpha$ to $x m \triangleright f:: M \beta$, in other words, to the operator section $(\triangleright f)$. In this notation, the associative law (3) becomes the equation $g^{*} \cdot f^{*}=\left(g^{*} \cdot f\right)^{*}$.

The following definition generalises the notion of monad by identifying for each object $x \in \mathcal{X}$ not just an object $M x \in \mathcal{X}$ in the original category, but an object $F x \in \mathcal{A}$ in another category $\mathcal{A}$ that is related to $\mathcal{X}$ be a forgetful functor $U: \mathcal{A} \rightarrow \mathcal{X}$.

Definition 1 If $\mathcal{A}$ and $\mathcal{X}$ are categories with a functor $U: \mathcal{A} \rightarrow \mathcal{X}$, then we define an $\mathcal{A}$-monad on $\mathcal{X}$ to be a triple $\left\langle F,{ }_{\sharp}^{\sharp}, \eta\right\rangle$ that assigns

- an object $F x \in \mathcal{A}$ to each $x \in \mathcal{X}$,

- an arrow $f^{\sharp}: F x \rightarrow F y$ in $\mathcal{A}$ to each arrow $f: x \rightarrow U F y$ in $\mathcal{X}$, and

- an arrow $\eta_{x}: x \rightarrow U F x$ to each object $x \in \mathcal{X}$,

in such a way that

$$
\begin{aligned}
\eta_{x}^{\sharp} & =\operatorname{id}_{F x}, \\
U f^{\sharp} \cdot \eta_{x} & =f,
\end{aligned}
$$

and if $f: x \rightarrow U F y$ and $g: y \rightarrow U F z$ then

$$
g^{\sharp} \cdot f^{\sharp}=\left(U g^{\sharp} \cdot f\right)^{\sharp} .
$$

A few immediate remarks are in order:

1. The concept of an $\mathcal{A}$-monad generalises that of a monad on $\mathcal{X}$ in two ways. Firstly, each $\mathcal{A}$-monad induces a simple monad $\left\langle T,{ }_{-}{ }^{*}, \eta\right\rangle$ on $\mathcal{X}$, where $T x=U F x$ and if $f: x \rightarrow T y$ then $f^{*}=U\left(f^{\sharp}\right)$. It is a matter of routine to verify that the requisite equations hold in this monad. Secondly, if we set $\mathcal{A}=\mathcal{X}$ and set $U$ to be the identity functor $\operatorname{Id} \mathcal{X}: \mathcal{X} \rightarrow \mathcal{X}$, then the concept of an $\mathcal{A}$-monad reduces to that of an ordinary monad on $\mathcal{X}$.

2. Just as ordinary monads can be defined equationally in terms of functors and natural transformations, so the concept of an $\mathcal{A}$-monad can be presented equationally. Given the data in the definition above, we can extend $F$ to a functor by defining its action on an arrow $f: x \rightarrow y$ to be $F f=\left(\eta_{y} \cdot f\right)^{\sharp}: F x \rightarrow F y$. The family of arrows $\eta_{x}$ then becomes a natural transformation $\eta: \operatorname{Id}_{\mathcal{X}} \doteq U F$. Moreover, we can define a natural transformation $\nu: F U F \stackrel{\bullet}{\rightarrow}$ by $\nu_{x}=\mathrm{id}_{U F x}^{\sharp}$, and the three equations above then give the following three equations relating $\eta$ and $\nu$ :

$$
\begin{aligned}
\nu \cdot F U \nu & =\nu \cdot \nu U F, \\
\nu \cdot F \eta & =\mathrm{id}_{F}, \\
U \nu \cdot \eta U F & =\mathrm{id}_{U F} .
\end{aligned}
$$

(see Figure 1). These are slightly twisted copies of the equations in the equational definition of an ordinary monad.

Conversely, a translation the other way is obtained by setting $f^{\sharp}=\nu_{y} \cdot F f$ for each $f: x \rightarrow U F y$ in $\mathcal{X}$. The laws defining an $\mathcal{A}$-monad then follow from the three equations 

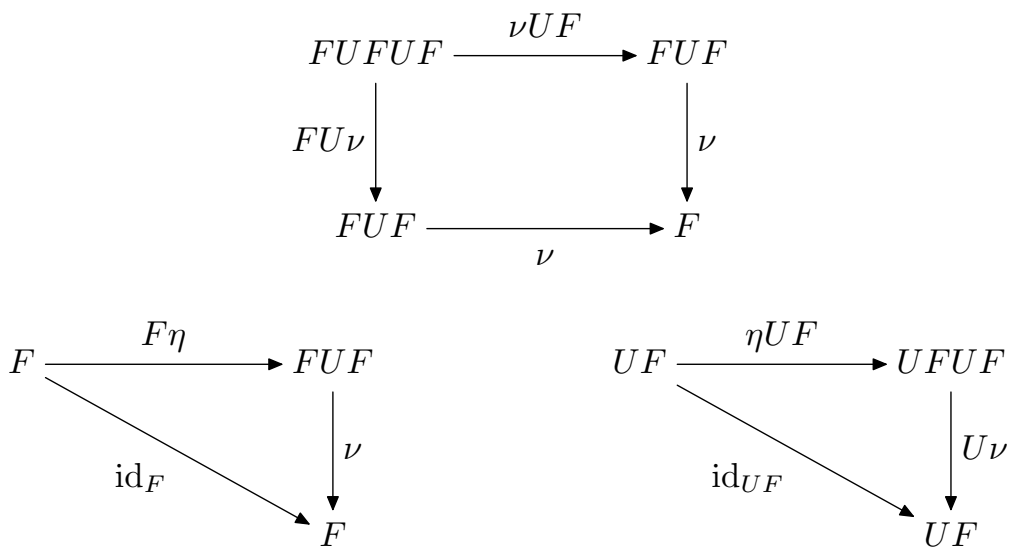

FIGURE 1: Laws for an $\mathcal{A}$-monad

just stated; in particular, the associative law (12) follows because

$$
\begin{aligned}
g^{\sharp} \cdot f^{\sharp} & =\nu_{z} \cdot F g \cdot \nu_{y} \cdot F f=\nu_{z} \cdot \nu_{U} F z \cdot F U F g \cdot F f \\
& =\nu_{z} \cdot F U \nu_{z} \cdot F U F g \cdot F f=\nu_{z} \cdot F\left(U\left(\nu_{z} \cdot F g\right) \cdot f\right)=\left(U g^{\sharp} \cdot f\right)^{\sharp} .
\end{aligned}
$$

3. An $\mathcal{A}$-monad is more than a monad on $\mathcal{X}$, but it is less than an adjunction between $\mathcal{X}$ and $\mathcal{A}$. In particular, a forgetful functor $U: \mathcal{A} \rightarrow \mathcal{X}$ can have (up to isomorphism) at most one right adjoint, but as we shall see, there may be multiple non-isomorphic $\mathcal{A}$-monads on $\mathcal{X}$. The key difference between the data given above and those that determine an adjunction is that the _ $\sharp$ operation is restricted to arrows $f: x \rightarrow U F y$, and does not apply to all arrows $f: x \rightarrow U a$ for any $a \in \mathcal{A}$.

We define morphisms of $\mathcal{A}$-monads as follows:

Definition 2 If $\left\langle F,{ }_{-}^{\sharp}, \eta\right\rangle$ and $\left\langle F^{\prime},{ }_{-}^{b}, \eta^{\prime}\right\rangle$ are $\mathcal{A}$-monads on $\mathcal{X}$, then a morphism $\theta$ between them is a family of arrows $\theta_{x}: F x \rightarrow F^{\prime} x$ in $\mathcal{A}$ for each $x \in \mathcal{X}$, such that whenever $f: x \rightarrow U F y$ in $\mathcal{X}$,

$$
\begin{aligned}
U \theta_{x} \cdot \eta_{x} & =\eta_{x}^{\prime} \\
\theta_{y} \cdot f^{\sharp} & =\left(U \theta_{y} \cdot f\right)^{b} \cdot \theta_{x} .
\end{aligned}
$$

Equivalently, in terms of functors and natural transformations, we may define a morphism of $\mathcal{A}$ monads to be a natural transformation $\theta: F \stackrel{\bullet}{\rightarrow} F^{\prime}$ such that $U \theta \cdot \eta=\eta^{\prime}$ and $\theta \cdot \nu=\nu^{\prime} \cdot(\theta \star U \star \theta)$, where $\nu^{\prime}: F^{\prime} U F^{\prime} \dot{\hookrightarrow} F^{\prime}$ and $\star$ denotes horizontal composition of natural transformations, so that $\theta \star U \star \theta=\theta U \cdot \theta=\theta \cdot U \theta$. In either style, the obvious definitions of identity morphisms and composition of morphisms make the class of $\mathcal{A}$-monads on $\mathcal{X}$ into a category. If $\theta: F \stackrel{\bullet}{\rightarrow} F^{\prime}$ is any

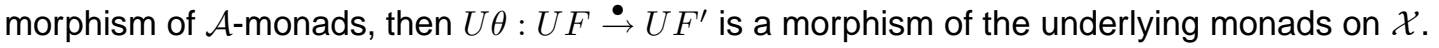

For ordinary monads, it is well known that an adjunction between $\mathcal{X}$ and $\mathcal{A}$ creates a monad on $\mathcal{X}$, and it seems reasonable to expect that it should create an $\mathcal{A}$-monad also. In fact, this $\mathcal{A}$-monad is an initial object in the category:

Lemma 1 Let $\langle F, U, \eta, \epsilon\rangle: \mathcal{X} \rightarrow \mathcal{A}$ be an adjunction, and let $\nu=\epsilon F$. Then $\langle F, \eta, \nu\rangle$ is an initial object in the category of $\mathcal{A}$-monads.

Proof: We first show that $\langle F, \eta, \nu\rangle$ is an $\mathcal{A}$-monad. Equation (13) follows because $\epsilon$ is a natural transformation:

$$
\nu \cdot F U \nu=\epsilon F \cdot F U \epsilon F=\epsilon F \cdot \epsilon F U F=\nu \cdot \nu U F .
$$

Equations (14) and (15) are instances of the two triangle laws of the adjunction:

$$
\nu \cdot F \eta=\epsilon F \cdot F \eta=\operatorname{id}_{F},
$$


and

$$
U \nu \cdot \eta U F=U \epsilon F \cdot \eta U F=\mathrm{id}_{U F} .
$$

Now suppose $\left\langle F^{\prime}, \eta^{\prime}, \nu^{\prime}\right\rangle$ is any $\mathcal{A}$-monad, and define a morphism $\theta: F \dot{\hookrightarrow} F^{\prime}$ by $\theta=\epsilon F^{\prime} \cdot F \eta^{\prime}$. This does give a morphism, for

$$
U \theta \cdot \eta=U \epsilon F^{\prime} \cdot U F \eta^{\prime} \cdot \eta=U \epsilon F^{\prime} \cdot \eta U F^{\prime} \cdot \eta^{\prime}=\eta^{\prime},
$$

and

$$
\begin{aligned}
& \nu^{\prime} \cdot(\theta \star U \star \theta)=\nu^{\prime} \cdot \theta U F^{\prime} \cdot F U \theta=\nu^{\prime} \cdot \epsilon F U F^{\prime} \cdot F \eta^{\prime} U F^{\prime} \cdot F U \theta \\
& \quad=\epsilon F^{\prime} \cdot F U \nu^{\prime} \cdot F \eta^{\prime} U F^{\prime} \cdot F U \theta=\epsilon F^{\prime} \cdot i d_{F U F^{\prime}} \cdot F U \theta=\theta \cdot \epsilon F=\theta \cdot \nu
\end{aligned}
$$

Lastly, suppose $\theta_{1}: F \stackrel{\bullet}{\rightarrow} F^{\prime}$ is another morphism. We can calculate,

$$
\theta=\epsilon F^{\prime} \cdot F \eta^{\prime}=\epsilon F^{\prime} \cdot F U \theta_{1} \cdot F \eta=\theta_{1} \cdot \epsilon F \cdot F \eta=\theta_{1} .
$$

So $\theta$ is a unique morphism from $F$ to $F^{\prime}$.

While an adjunction between $\mathcal{X}$ and the whole of the category $\mathcal{A}$ gives an initial object in the category of $\mathcal{A}$-monads, other $\mathcal{A}$-monads can be obtained from adjunctions between $\mathcal{X}$ and subcategories of $\mathcal{A}$. If $\left\langle F, U^{\prime}, \eta, \epsilon\right\rangle: \mathcal{X} \rightarrow \mathcal{B}$ for some subcategory $\mathcal{B} \subseteq \mathcal{A}$ (where $U^{\prime}=\left.U\right|_{\mathcal{B}}$ is the restriction of $U$ ), then we can define $\nu=\epsilon F$ and obtain an $\mathcal{A}$-monad $\langle F, \eta, \nu\rangle$ as before. The converse of this result also holds: given any $\mathcal{A}$-monad, we can find an adjunction between $\mathcal{X}$ and a subcategory of $\mathcal{A}$ that induces it in this way.

Lemma 2 If $\langle F, \eta, \nu\rangle$ is an $\mathcal{A}$ monad, then there is a subcategory $\mathcal{B}$ of $\mathcal{A}$ and an adjunction $\left.\left\langle F, U^{\prime}, \eta, \epsilon\right\rangle\right): \mathcal{X} \rightarrow \mathcal{B}$ such that $\nu=\epsilon F$.

Proof: Let $\mathcal{B}$ be the full subcategory of $\mathcal{A}$ that contains the objects $F x$ for $x \in \mathcal{X}$, and define $U^{\prime}=\left.U\right|_{\mathcal{B}}$ and $\epsilon_{a}=\nu_{x}$ where $a=F x$. (If $F x=F y$, then it follows that $\nu_{x}=\nu_{y}$ because $\nu: F U F \stackrel{\bullet}{\rightarrow} F$ is a natural transformation.) The required equations for the adjunction follow from the defining equations of the $\mathcal{A}$-monad:

$$
\epsilon F \cdot F \eta=\nu \cdot F \eta=\operatorname{id}_{F},
$$

and if $a=F x$ then

$$
U \epsilon_{a} \cdot \eta_{U a}=U \nu_{x} \cdot \eta_{U F x}=\operatorname{id}_{U F x}=\mathrm{id}_{U a} .
$$

This completes the proof.

\section{APPLICATION TO COMBINATORIAL SEARCH}

The general picture of the category of $\mathcal{A}$-monads can now be applied to the particular case of search strategies. For the moment, we will simplify the discussion by restricting attention to finite search spaces, and ignore the problem of modelling programs where the search space is infinite. That restriction will be removed in Section 6.

A search strategy is a monad equipped with the operations $\oplus$, zero and wrap and satisfying the equations given in Section 2. This makes it appropriate to take $\mathcal{A}$ to be the category of $(\Omega, E)$ algebras and homomorphisms, where $\Omega$ is the graded set of operators $\left\{\oplus_{2}, \operatorname{zero}_{0}\right.$, wrap $\left._{1}\right\}$, and $E$ is the set of equations,

$$
\{(x \oplus y) \oplus z=x \oplus(y \oplus z), \text { zero } \oplus x=x=x \oplus \text { zero }\} .
$$

A standard result states that there is an adjunction between the category of sets and the category of $(\Omega, E)$-algebras that gives the free algebra generated by any set. Lemma 1 of Section 4 then tells us that the $\mathcal{A}$-monad containing these free algebras is an initial object in its category. 

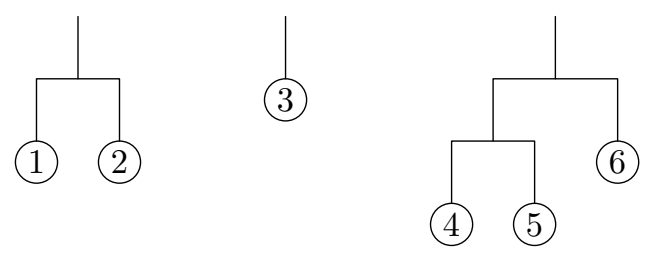

FIGURE 2: A typical forest

For our $\Omega$ and $E$, this free algebra is the set of forests with leaves from the given set. A typical element where the leaves are integers is the forest

$$
\text { [Fork [Tip 1, Tip 2], Tip 3, Fork [Fork [Tip 4, Tip 5], Tip 6]] }
$$

shown in Figure 2. Writing $w$ for wrap and $\{x\}$ for return $x$, this can be expressed in terms of the operations as

$$
w(\{1\} \oplus\{2\}) \oplus\{3\} \oplus w(w(\{4\} \oplus\{5\}) \oplus\{6\}) .
$$

In this case, it should be clear that the type of forests, together with the operations defined in Section 2, represents exactly the set of expressions of this form, and that distinct expressions give distinct values in the type. The results of Section 4 then tell us that this type can be made into an $\mathcal{A}$-monad, and it remains only to calculate a definition of the binding operator $\triangleright$. Any forest $x f$ can be written in the form

$$
x f=\left[t_{1}\right]+\left[t_{2}\right]+\ldots+\left[t_{n}\right]
$$

and then we see that

$$
x f \triangleright f=\left(\left[t_{1}\right] \triangleright f\right)++\left(\left[t_{2}\right] \triangleright f\right)+\ldots++\left(\left[t_{n}\right] \triangleright f\right),
$$

so $x f \triangleright f=\operatorname{concat}(\operatorname{map}(\operatorname{bin} d t f) x f)$, where bindt $f t=[t] \triangleright f$. Now we can calculate that

$$
\text { bindt } f(\text { Leaf } x)=f x,
$$

since $[$ Leaf $x]=$ return $x$, and bindt $f($ Fork $x f)=[$ Fork $(x f \triangleright f)]$, since $[$ Fork $x f]=$ wrap $x f$. In this way, we can derive the definition of $\triangleright$ from the algebraic laws that characterise a bunch, something we already did in Section 2 for the bunch of breadth-first search.

The part played in this calculation by the results developed in Section 4 needs to be explained. We have shown that any adjunction of the kind created by an algebraic theory generates an $\mathcal{A}$ monad, so that there exists an operation $\triangleright$ that satsifies all the required laws. What we have just shown is that any operation that satisfies the laws must also satisfy the equations that make up a recursive definition of $\triangleright$. Since this recursive definition describes a unique operation, we may be sure that this operation is the required binding operator for the monad.

We can now play a game like the one that leads to the "Boom hierarchy" trees-lists-bags-sets of collection types: by adding equations to the algebraic specification, we obtain alternative models that identify previously distinct terms. For each set of equations $E^{\prime}$ that extends our original set $E$, we obtain a full subcategory of the category of $(\Omega, E)$-algebras, containing just those that actually satisfy the equations $E^{\prime}$, together with an adjunction between sets and the subcategory. This gives an $\mathcal{A}$-monad on sets, and in particular a unique definition of the binding operator $\triangleright$ for the resulting notion of search.

For example, if we put wrap $x=x$, this reduces the example term to

$$
\{1\} \oplus\{2\} \oplus\{3\} \oplus\{4\} \oplus\{5\} \oplus\{6\},
$$

i.e., to something that can be represented by the list $[1,2,3,4,5,6]$, and we obtain depth-first search. Again, once we see that the expressions in this normal form are faithfully represented by lists, it follows that there is an A-monad based on lists, and the definition of the binding operator can be calculated. 
Alternatively, we may add the equation that $\operatorname{wrap}$ distributes over $\oplus$, reducing the example term to

$$
w(\{1\}) \oplus w(\{2\}) \oplus\{3\} \oplus w(w(\{4\})) \oplus w(w(\{5\})) \oplus w(\{6\}) .
$$

With these equations, any finite term can be reduced to a normal form that is a sum of terms, each of the form $w^{n}(\{x\})$; such a normal form reveals the left-to-right order of the solutions and the depth in the tree at which each one appears, without preserving the branching structure that links the solutions to roots in the forest. Thus it corresponds to the bunch of depth-bounded search.

Adding the further equation that wrap is commutative lets us group together the terms $w^{n}(\{x\})$ that have the same depth $n$, and reduces our example term to

$$
\{3\} \oplus w(\{1\} \oplus\{2\} \oplus\{6\}) \oplus w(w(\{4\} \oplus\{5\})) .
$$

In general, the normal form is a sum of terms $w^{n}(s)$ for distinct $n$, where $s$ is itself a sum of singletons in an arbitrary order. This corresponds closely to breadth-first search, where we may discover the bag of solutions that occur at each depth in the tree. We remarked earlier that it was impossible to get an associative binding operator for breadth-first search without making $\oplus$ commutative, and so treating each level of the result as a bag rather than a list. Here we see that imposing commutativity has the positive effect of simplifying the normal forms and leading us directly to the stream-of-bags representation.

If we accept that the type containing lists of bags is a proper representation of the normal forms just described, then the calculation in Section 2 that led to a definition of $\triangleright$ is correct, and the operator it defines satisfies all the required laws, including the associative law (3). This is reassuring, since proving the associative law directly is rather difficult (see [5]).

In addition to the notion of an $\mathcal{A}$-monad, our categorical approach points us the the notion of a morphism of $\mathcal{A}$-monads, a parametric system of homomorphisms of algebras that is also a morphism of monads. For our initial object, there is a unique such morphism to any other bunch $M$, and we can easily derive a definition of this morphism as a functional program. It gives the bunch object that results from searching any forest:

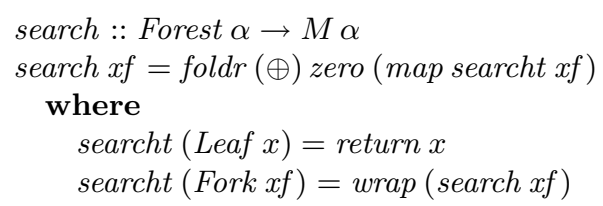

The fact that search is a bunch morphism reassures us that for any bunch $M$, the same results are obtained by running a program that builds a forest and then searching it as are obtained by running the same program directly in $M$.

Jeremy Gibbons and Geraint Jones have shown [7] that the familiar implementations of depthfirst and breadth-first search that use a stack and a queue respectively can be derived from this definition of search by recasting it as an unfold.

\section{INFINITE SEARCH SPACES}

The discussion of the previous section indicates that, at least for finite searches, all compositional search strategies can be made part of a single algebraic framework. It would be nice to do the same for infinite searches, and preferable to do it in a way that reflects the fairness of strategies like breadth-first search. One possible approach is based on the theory of metric spaces.

Let us define a fair bunch to be a bunch $M$ such that type $M \alpha$ is also a complete bounded metric space under some metric $d$ (see, for example, [8]), subject to certain restrictions. Our first example will be the bunch of forests, where we now allow infinite trees, provided that they remain finitely branching. There we can define $d\left(f_{1}, f_{2}\right)=2^{-n}$, where $n$ is the first level at which $f_{1}$ and $f_{2}$ differ, or $d\left(f_{1}, f_{2}\right)=0$ if $f_{1}=f_{2}$. More precisely, we can define a function 
prune $:$ Int $\rightarrow$ Forest $\alpha \rightarrow$ Forest $\alpha$

that cuts off all branches in a forest below a specified depth; we then look for the smallest $n$ such that prune $n f_{1} \neq$ prune $n f_{2}$.

The operations of the bunch are related to this metric in a number of ways:

1. The function wrap is a contraction, in the sense that then $d\left(\right.$ wrap $f_{1}$, wrap $\left.f_{2}\right) \leq \rho d\left(f_{1}, f_{2}\right)$ for some $\rho<1$; actually, we can take $\rho=1 / 2$.

2. The operation $\oplus$ satisfies $d\left(f_{1} \oplus f_{2}, f_{1}^{\prime} \oplus f_{2}^{\prime}\right) \leq \max \left(d\left(f_{1}, f_{1}^{\prime}\right), d\left(f_{2}, f_{2}^{\prime}\right)\right)$.

3. The finite elements of $M \alpha$-those that are the value of some finite term in the operations - are dense in $M \alpha$, so that every element of $M \alpha$ is the limit of some sequence of finite elements.

The first two of these properties are sufficient to guarantee that any guarded recursive definition has a unique solution. By a guarded recursion, I mean an equation of the form $t=F(t)$, where $F(x)$ is defined by a term where each occurrence of $x$ is 'guarded' by at least one application of wrap. Standard techniques allow this result to be extended to recursive definitions of functions of the form $f(x)=F(f(g(x)))$ where $F$ is guarded. The third property ensures that any continuous function from $M \alpha$ to some other complete metric space is uniquely determined by its behaviour on the finite elements.

Of the other bunches, we cannot hope to make a fair bunch from depth-first search, because the wrap operation there is the identity function, and that cannot be a contraction. The lack of a general result about the existence of free continuous algebras of this kind means that we must also treat the other bunches one at a time.

For breadth-first search, we can define the distance between two streams of bags $m_{1}$ and $m_{2}$ to be $d\left(m_{1}, m_{2}\right)=2^{-n}$, where $n$ is smallest such that take $n m_{1} \neq$ take $n m_{2}$. Similarly, for depthbounded search we may take $d\left(p_{1}, p_{2}\right)=2^{-n}$ where $n$ is smallest such that $p_{1} n \neq p_{2} n$.

Though considerations of space prevent a full development of the relevant theory here, we can give an outline of it. The appropriate notion of morphism for fair bunches is a family of uniformly continuous functions that is also a morphism of the underlying bunches. In fact, all the functions we need to consider in the existing examples are actually Lipschitz continuous.

Using uniform continuity assures is that the image of a Cauchy sequence is again a Cauchy sequence, and allows us to extend functions defined on finite elements to cover infinite elements too, in a way that is unique because we assume the finite elements are dense. This allows us to extend the definitions of the operations from finite to infinite elements in a way that preserves the algebraic laws, and gives us a unique morphism from the fair bunch of forests to any other fair bunch.

\section{CONCLUSION}

We have seen that in functional programming, several different strategies for combinatorial search can be made part of the same algebraic framework, one that emphasizes the compositional nature of the strategies. This algebraic framework adds one operation wrap to the standard MonadPlus type-class of Haskell, in effect making it possible for a computation to signal that it has produced all the results with a specified cost. We have shown, at least for finite search spaces, that these different search strategies all arise by adding various equations to an algebraic theory that describes the set of outcomes of the search, and we have suggested a way in which the theory could be extended to cover infinite search spaces also.

The search strategies we have covered are all oblivious, in the sense that they depend only on the depth of solutions in the tree of choices, and are not guided by any heuristic estimate of distance from a solution. It would be interesting to see whether the present framework could be extended 
to include a (real-valued) notion of distance, so that strategies such as best-first and $A^{*}$ search could also be covered.

Many others have written about implementations of combinatorial search in higher-order functional programming, and it is appropriate to mention some recent related work here. Much of this work focusses on implementations of depth-first search. Ralf Hinze [9] has shown that an implementation of depth-first search using success and failure continuations can be more efficient in Haskell that one based on lazy streams. He also considers the algebraic laws that are satisfied by our combinators and by others that correspond to Prolog's control structures, including cut. Kiselyov, Shan, Friedman and Sabry [6] extend this work to show how fair interleaving can also be implemented, though with the failure of associativity that we noted earlier. In an elegant paper, Wand and Vaillancourt [10] use operational semantics to show the equivalence of the continuation-based and stream-based models of depth-first search.

\section{ACKNOWLEDGEMENTS}

The author is happy to acknowledge that part of this work was carried out while he held a visiting chair at the University of New South Wales, and thanks Carroll Morgan for his generous hospitality and helpful suggestions.

\section{REFERENCES}

[1] S. J. Russell and P. Norvig. Artificial Intelligence: A Modern Approach, 2nd ed. Prentice Hall, 2003.

[2] L. G. L. T. Meertens. Algorithmics-Towards programming as a mathematical activity. In J. W. de Bakker, M. Hazewinkel, and J. K. Lenstra, eds., Proc. of CWI Symposium on Mathematics and Computer Science. vol. 1 of CWI Monographs, pp. 289-334. North-Holland Publ. Co., 1986.

[3] R. S. Bird. An introduction to the theory of lists. In M. Broy, ed., Calculi of Discrete Design, vol. 36 of NATO ASI Series F, pp. 5-42. Springer-Verlag, 1987.

[4] J. M. Spivey and S. Seres. Combinators for logic programming. In J. Gibbons and O. de Moor, eds., The Fun of Programming, Cornerstones of Computing, pp. 177-200. Palgrave MacMillan, 2003.

[5] J. M. Spivey. Combinators for breadth-first search. J. of Funct. Program., 10(4), 397-408, 2000.

[6] O. Kiselyov, C.-c. Shan, D. P. Friedman, and A. Sabry. Backtracking, interleaving and terminating monad transformers. In Proc. of 10th ACM SIGPLAN Int. Conf. on Functional Programming, ICFP 2005 (Tallinn, Sept. 2005), pp. 192-203. ACM Press, 2005. Also in ACM SIGPLAN Notices, 40(9), 192-203, 2005.

[7] J. Gibbons and G. Jones. The under-appreciated unfold. In Proc. of 3rd ACM SIGPLAN Int. Conf. on Functional Programming, ICFP '98 (Baltimore, MD, Sept. 1998), pp. 273-279. ACM Press, 1998. Also in ACM SIGPLAN Notices, 34(1), 273-279, 1999.

[8] W. A. Sutherland. Introduction to Metric and Topological Spaces. Oxford University Press, 1975.

[9] R. Hinze. Prolog's control constructs in a functional setting-Axioms and implementation. Int. J. on Found. of Comput. Sci., 12(2), pp. 125-170, 2001.

[10] M. Wand and D. Vaillancourt. Relating models of backtracking. In Proc. of 9th ACM SIGPLAN Int. Conf. on Functional Programming, ICFP 2004 (Snowbird, UT, Sept. 2004), pp. 54-65. ACM Press, 2004. Also in ACM SIGPLAN Notices, 39(9), 54-65, 2004.

\section{APPENDIX: NOTATION}

We use an infix dot for composition of arrows $g \cdot f$, and also for 'vertical' composition of natural

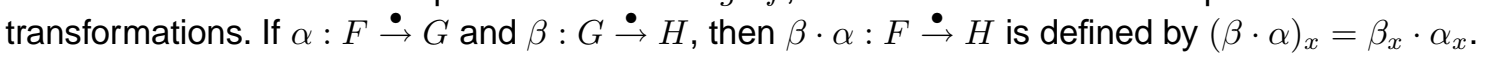

Composition of functors with functors and with natural transformations is denoted by juxtaposition. As examples of the latter, we if $\alpha: F \dot{\rightarrow} F^{\prime}$ and $\beta: G \dot{\rightarrow} G^{\prime}$ for functors $F, F^{\prime}: \mathcal{X} \rightarrow \mathcal{Y}$ and 


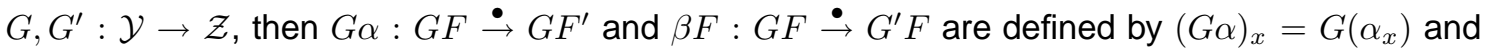
$(\beta F)_{x}=\beta_{F x}$.

'Horizontal' composition of natural transformations is denoted by $\star$ : if $\alpha$ and $\beta$ are as above, then $\beta \star \alpha: G F \stackrel{\bullet}{\rightarrow} G^{\prime} F^{\prime}$ is defined by $\beta \star \alpha=\beta F^{\prime} \cdot G \alpha=G^{\prime} \alpha \cdot \beta F$. This composition is associative, and as a special case, if $\theta: F \doteq F^{\prime}$ for $F: \mathcal{X} \rightarrow \mathcal{A}$ and $U: \mathcal{A} \rightarrow \mathcal{X}$, we write $\theta \star U \star \theta$ for the natural transformation $\phi=\theta \star \operatorname{Id}_{U} \star \theta: F U F \rightarrow F^{\prime} U F^{\prime}$ with $\phi_{x}=\theta_{U F^{\prime} x} \cdot F U \theta_{x}=F^{\prime} U \theta_{x} \cdot \theta_{U F x}$. 